\title{
Cervicovaginal Microbiome Factors in Clearance of Human Papillomavirus Infection
}

\author{
Wenkui Dai ${ }^{1,2,3 \dagger}$, Hui $\mathrm{Du}^{1,2,3 \dagger}$, Shuaicheng $\mathrm{Li}^{4 *}$ and Ruifang $\mathrm{Wu}^{1,2,3 *}$ \\ 1 Department of Obstetrics and Gynecology, Peking University Shenzhen Hospital, Shenzhen, China, ${ }^{2}$ Institute of Obstetrics \\ and Gynecology, Shenzhen Peking University-The Hong Kong University of Science and Technology (PKU-HKUST) Medical \\ Center, Shenzhen, China, ${ }^{3}$ Shenzhen Key Laboratory on Technology for Early Diagnosis of Major Gynecologic Diseases, \\ Shenzhen, China, ${ }^{4}$ Department of Biomedical Engineering, City University of Hong Kong, Hong Kong, China
}

Persistent high-risk human papillomavirus (hrHPV) infection is the highest risk to cervical cancer which is the fourth most common cancer in women worldwide. A growing body of literatures demonstrate the role of cervicovaginal microbiome (CVM) in hrHPV susceptibility and clearance, suggesting the promise of CVM-targeted interventions in protecting against or eliminating HPV infection. Nevertheless, the CVM-HPV-host interactions are largely unknown. In this review, we summarize imbalanced CVM in HPV-positive women, with or without cervical diseases, and the progress of exploring CVM resources in HPV clearance. In addition, microbe- and host-microbe interactions in HPV infection and elimination are reviewed to understand the role of CVM in remission of HPV infection. Lastly, the feasibility of CVM-modulated and-derived products in promoting HPV clearance is discussed. Information in this article will provide valuable reference for researchers interested in cervical cancer prevention and therapy.

Keywords: cervical cancer, high-risk HPV, CVM-targeted intervention, CVM-derived product, HPV clearance

\section{INTRODUCTION}

Persistent high-risk human papillomavirus (hrHPV) infection is the highest risk to invasive cervical cancer (ICC), which has caused an estimated 570,000 new cases and 311,000 deaths in 2018 (1). Prophylactic vaccines are effective in preventing HPV infection, but providing limited protection against pre-existing HPV infection which impact large populations in developing countries for a long-lasting period $(2,3)$. It will be an imperative alternative to prevent HPV-infected cervical intraepithelial neoplasia (CIN) and ICC by eliminating HPV infection. An increasing number of literatures suggests the association of natural HPV clearance and CIN regression with cervicovaginal microbiome (CVM) (4-8), which modulate a finely-tuned immune responses balancing reproductive tolerance with protection against genital infections (9). Our and other studies demonstrated predominance of one or few Lactobacillus species in CVM of healthy lower reproductive tract (LRT), including Lactobacillus crispatus (community-state type I, CST I), Lactobacillus gasseri (CST II), Lactobacillus iners (CST III) and Lactobacillus jensenii (CST V) (10-14). These Lactobacillus species benefit reproductive health by inhibiting pathogens via produced bacteriocins, lactic acid and hydrogen peroxide (15). 
Emerging reports demonstrate imbalanced CVM in women with HPV infection, including increased bacterial diversity, depletion of Lactobacillus as well as identified high rate of natural HPV clearance in women with predominant $L$. crispatus in CVM $(4,8,16-21)$. To the best of our knowledge, there is no public report investigating the mechanism of interaction between HPV and microbiome, due to difficulties to cultivate HPV in vitro and limited mouse models for HPVmedicated cervical dysplasia or cancer. Nevertheless, a number of studies support the concept that CVM modulates immune microenvironment through microbe- or microbe-host interactions to impact the risk of viral infections and clearance (9, 22-25). For instance, Lactobacillus conferred colonization resistance to Gardnerella vaginalis which induced suppressive immune responses beneficial to persistent HPV infection (22). M $\mathrm{N}$ Anahtar et al. demonstrated that CVM was the main modulator of immune responses in lower reproductive tract (LRT) and affected the risk of human immunodeficiency virus (HIV) infection (23). Peptidoglycans (PGN) produced by isolated vaginal $L$. crispatus activate Langerhans cells (LCs), which is the most important antigen presenting cells (APCs) in cervical epithelium (25), and several follow-up investigations further suggest a strong in vivo relationship between LCs activities and HPV clearance (26-28).

In this review, we first summarize the association of CVM with HPV infection and clearance, then discuss mechanisms of microbiome, host responses and HPV interaction. Lastly, several potentials are explored about how to eliminate pre-existing HPV infection via microbiome-derived products or microbiometargeted interventions.

\section{IMBALANCED CVM IN HPV INFECTION}

Emerging evidence suggests association between CVM and HPV infection and persistence. Almost all cross-sectional studies consistently found higher diversity of CVM in HPV-positive women, with or without CIN, as compared to HPV-negative individuals (16-21, 29-32). In recent decade, a growing body of literature suggests that depletion of Lactobacillus and overgrowth of anaerobic bacteria is associated with increased CVM diversity (Figure 1) (16-21, 29, 30). For individuals infected with HPV but without CIN or ICC, initial cross-sectional studies involving Korean ( $\mathrm{n}=68$ selected from 912 women in Healthy Twin Study) and Chinese $(n=70)$ women identified reduced levels of Lactobacillus as well as higher abundance of bacterial vaginitis (BV)-associated bacteria such as Gardnerella, Sneathia and Megasphaera $(18,30)$. This is consistent with increased susceptibility to HPV infection in women with BV revealed by meta-analysis (33). Besides to Gardnerella, Sneathia and Megasphaera, additional reports found greater relative abundance of Atopobium, Bacteroides, Prevotella and lower proportion of Lactobacillus in CVM of HPV-positive women $(16,17,29)$. Studies involving women with CIN or ICC consistently found significant decrease of Lactobacillus and substantial increase in CVM diversity compared with HPVnegative individuals $(18,19,31,32)$.

At species level of Lactobacillus, a marked decrease of $L$. crispatus was found in CVM of women with HPV infection, CIN or ICC, while L. iners-dominant CVM had higher risk of CIN (8, 18, 31, 32, 34). Additionally, women with HPV infection had accumulation of Bacteroides plebeius, Acinetobacter lwoffii, Prevotella buccae, Dialister invisus, G. vaginalis, Prevotella buccalis and Prevotella timonensis in $\operatorname{CVM}(29,31,32,34)$. For instance, a study involving 70 women with CIN and $50 \mathrm{HPV}$ negative women indicated that 6-fold risk of CIN associated with unique CVM, which is characterized by paucity of $L$. crispatus, enriched A. vaginae, G. vaginalis and L. iners (30). Two independent systematic reviews and meta-analysis also found that L. crispatus correlated with decreased risk of hrHPV infection and $\operatorname{CIN}(35,36)$. Compared with L. crispatusdominant CVM, women with non-Lactobacillus- or L. inersdominant CVM had 2-3 times higher odds of hrHPV prevalence and CIN, as well as 3-5 times higher odds of any prevalent HPV (95\% CI) (35).

Besides to microbial components, emerging literature explores functional difference of CVM between HPV-positive and HPV-negative women (37-39). Functional prediction of $16 \mathrm{~S}$ rDNA amplicon sequencing data found accumulation of multiple pathways in HPV-infected and CIN women, including those of folate biosynthesis and oxidative phosphorylation (37). Metagenomic analysis of 17 CIN, 12 ICC cases and 18 healthy individuals found enriched genes related to peptidoglycan synthesis as well as depletion of dioxin degradation and 4-oxalocrotonate tautomerase in CVM of women with CIN or ICC (38). Biofilm formation assessment identified higher formation rate in HPV-positive women (45\%) compared to HPV-negative women (21.9\%) (39), which may be

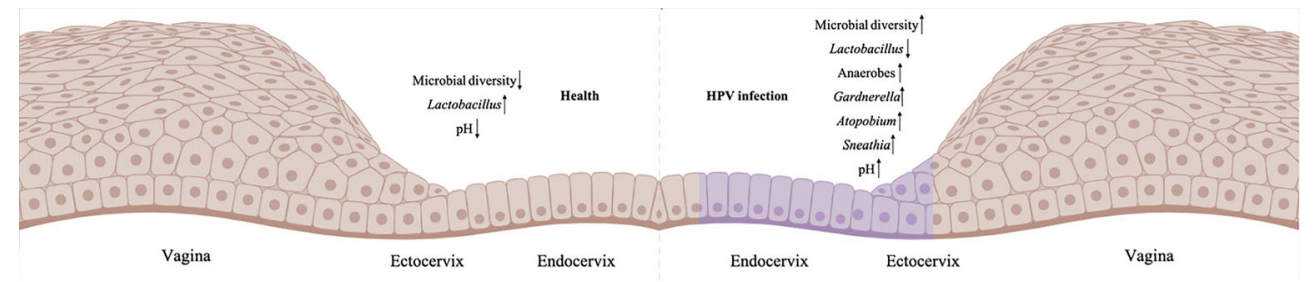

FIGURE 1 | Imbalanced CVM in HPV-infected cervix. The left is the normal cervicovaginal microenvironment without HPV infection, and the right is HPV-positive microenvironment (HPV-infected cells are labelled purple). Created in BioRender.com. 
attributed to increased levels of obligate anaerobic bacteria in CVM of HPV-infected women, such as G. vaginalis with sialidase-encoding gene involved in biofilm formation (8).

Above-mentioned observational studies are only possible to demonstrate association of CVM with HPV infection and CIN diseases rather than causality. Longitudinal data is increasingly applied to explore the causal link $(7,40,41)$, which has profound clinical impact to provide effective alternatives for therapeutic strategies of HPV-infected CIN. Six-month follow-up of 211 Nigerian women showed the association of Lactobacillus paucity and high CVM diversity with persistent hrHPV infection (40). Analysis of serial cervicovaginal specimens obtained over 8-10 years unraveled that high relative abundance of $L$. crispatus in CVM had the lowest risk of HPV infection compared to other types of CVM, according to 16S V1-V2 rRNA gene amplicon sequencing and HPV DNA testing conducted annually (41). Brotman and colleagues collected self-sampled mid-vaginal swabs twice a week for 16 weeks from 32 reproductive-age women, and showed that depletion of Lactobacillus in CVM may increase the chance to acquire transient and persistent HPV infection (7). Consistently, meta-analysis involving 39 articles suggests the protection against HPV infection imposed by Lactobacillus-dominant CVM (42). Another systematic review and meta-analysis of longitudinal studies also support a causal relationship between non-Lactobacillus-dominant CVM and cervical carcinogenesis via the effect of CVM on HPV infection (RR 1.33, 95\% CI) and persistence (RR 1.14) (43).

\section{CVM IS ASSOCIATED WITH NATURAL HPV CLEARANCE AND CIN REGRESSION}

According to a follow-up analysis on 55 women with HPV infection and 17 age-matched healthy HPV-negative women, $L$. crispatus was the most abundant Lactobacillus species in individuals with natural HPV clearance (Figure 2A) (8). Conversely, high proportion of Atopobium in CVM had significantly slowed HPV remission rate in 16-week follow-up, compared to L. crispatus-dominant $\mathrm{CVM}^{8}$. Another longitudinal study involving 64 HPV16-positive women found more frequent transition between identified CSTs, including dominant Lactobacillus sp., L. iners, two mixed non-Lactobacillus of CVM, in women with persistent HPV16 infection (34\% with averaged 155.5 days interval) when compared to women with natural clearance of HPV16 (19\% with averaged 162 days interval) (Figure 2A) (6). Consistently, Anita Mitra and partners found more stable CVM in women with CIN2 regression, as compared to individuals with CIN persistence or progression ${ }^{4}$. In this study,

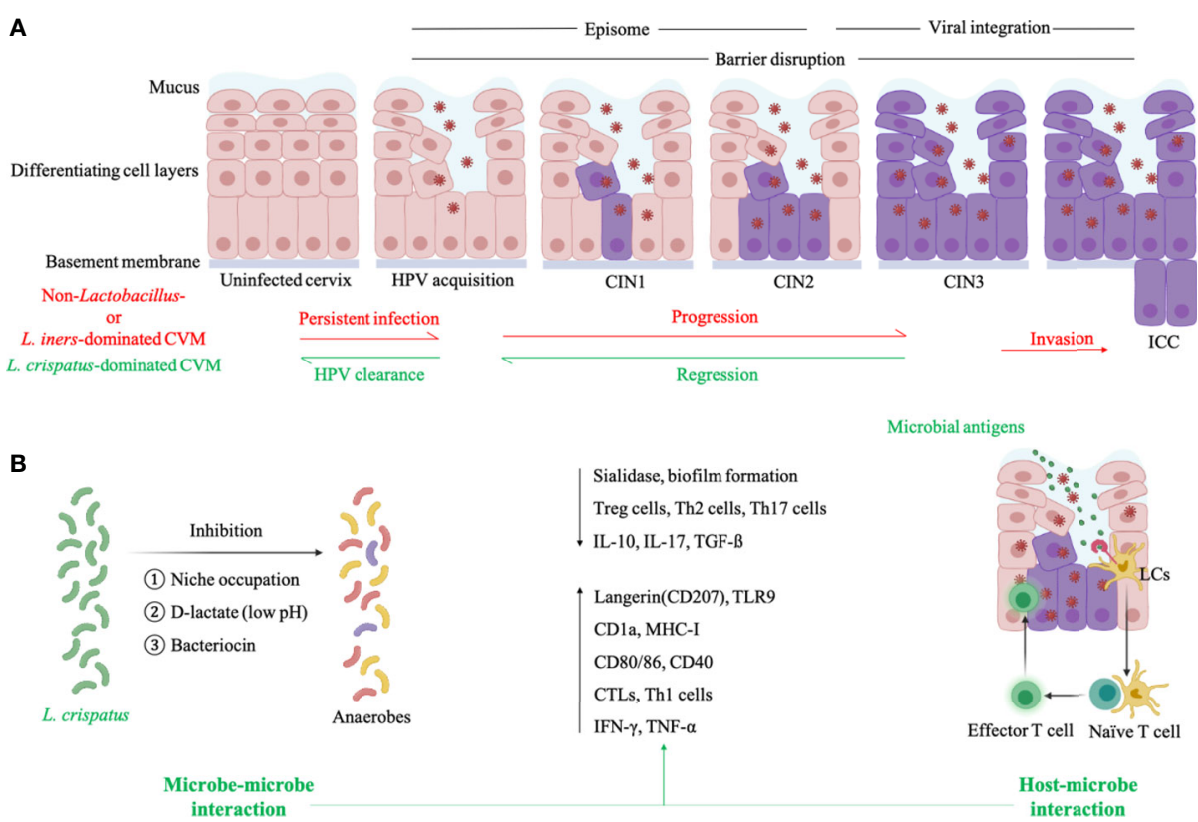

FIGURE 2 | Cervicovaginal microenvironment in persistent HPV infection and natural HPV clearance. (A) L. iners- or non-Lactobacillus-dominated CVM is characterized by allowing the proliferation of anaerobes which produce sialidase to disrupt epithelial barrier and then facilitate the entry of HPV. HPV particles exist in infected keratinocytes as episomes before entry into the nucleus, and viral integration induces high expression of E6/E7 proteins to promote abnormal cell proliferation as well as carcinogenesis. L. crispatus-dominated CVM is associated with natural HPV clearance and CIN regression. (B) For HPV-positive women with L. crispatus-dominated CVM, L. crispatus can inhibit growth of anaerobes through several mechanisms (microbe-microbe interaction) and activate LCs which is the only APCs in cervical epithelium to present HPV antigens and induce HPV-specific CMI (host-microbe interaction). Both microbe- and host-microbe interaction decrease the level of factors correlated with barrier impairment (sialidase and biofilm of anaerobes) and suppressive immunity (Treg, Th2, Th17 cells, IL-10, IL-17, TGF-B), and increase the expression of biomarkers in activated LCs (antigen-binding langerin and TLR9, antigen-presenting CD1a and MHC-I, co-stimulatory molecule CD80/86 and CD40) as well as CMI-associated molecules (cytotoxic T lymphocytes-CTLs, Th1 cells, IFN- $\gamma$, TNF- $\alpha$ ). This figure applied icons in BioRender.com. 
87 CIN2 patients aged 16-26 years old were included in two-year follow-up showing that women with Lactobacillus-dominant CVM at baseline are more likely to regress at 12 months while slower regression was associated with Lactobacillus depletion as well as increased abundance of Megasphaera, Prevotella timonensis and G. vaginalis (4). At species level, women with L. crispatusdominant CVM had faster regression and higher rate of CIN remission at 12 and 24 months (4).

A total of four CSTs, dominated by L. crispatus, L. iners, G. vaginalis and mixed genus, was identified in another study involving 273 women aged 18-25 years old (5). At first visit, Lactobacillus and Gardnerella abundance was associated with CIN2 regression and progression respectively. Second visit was conducted at least 305 days after first visit, and CIN2 progression had strong correlation with increased bacterial diversity. Functional prediction of $16 \mathrm{~S}$ rDNA amplicon sequencing data further showed the positive relationship between pathway of cell motility and CIN2 regression, while progression was in association with "Xenobiotics Biodegradation and Metabolism" pathway in CVM.

Fungal components in CVM were also associated with HPVinfected CIN regression (5). Mykhaylo Usyk and colleagues found the protective effect of fungal diversity against CIN progression (OR=0.90, 0.82-1.00) (5). Among fungus Candida, Malassezia and Sporidiobolaceae, the accumulation of Candida was identified in CVM of CIN1 which had the highest regression rate (5). Additionally, a retrospective investigation on 100,605 women who had 2 smears each over a period of 12 years, found that common fungus Candida in cervicovaginal microenvironment decreased the risk of squamous intraepithelial lesions (44).

\section{MICROBE- AND HOST-MICROBE INTERACTIONS IN HPV CLEARANCE}

The complexity of cervicovaginal microenvironment of women with HPV infection is determined by HPV, CVM and the host. To explore the contribution of CVM to promote or protect against HPV infection, there is much work to be done in exploring microbe-microbe interactions in CVM, as well as the interactions between microbe and HPV/host (Figure 2B).

Sialidase are a group of mucin-degrading enzymes produced by BV-associated G. vaginalis and Prevotella, and disrupt the integrity of mucosa as well as epithelium to aid the entry of HPV to basal keratinocytes (Figure 2A) (45). Besides to compromised cervical epithelial barrier, BV-associated anaerobes also impact several cellular pathways to enable persistent viral infection and subsequent disease development (46-50). Sneathia spp., commonly accumulated in CVM of BV and HPV-infected patients, belongs to Fusobacterium genus which can activate proinflammatory pathways and inhibit immunocytotoxicity to promote carcinogenesis (51). This information may explain the high susceptibility to HPV infection in women with BV and accumulation of vaginal obligate anaerobic bacteria in women with persistent HPV infection or cervical dysplasia progression (16-18, 29, 30, 33).
Vaginal Lactobacillus spp. can produce a large amount of lactic acid through glycogen fermentation, maintaining acidic environment to inhibit the colonization of several pathogenic species such as Chlamydia trachomatis, Neisseria gonorrhoeae and BV-associated G. vaginalis (Figure 2B) (15, 52-55). Bacteriocins produced by vaginal Lactobacillus also exhibit inhibitory effects on common pathogenic bacteria and certain fungi, such as G. vaginalis and Candida albicans (Figure 2B) (15, $56,57)$. In addition, Lactobacillus hold the potential to alter surface tension and thus bacterial adhesion which is pivotal in biofilm formation via excreted biosurfactants, therefore preventing overgrowth of pathogenic anaerobes, especially $G$. vaginalis (Figure 2B) (22, 58-60). Another defense factor derived from vaginal Lactobacillus is $\mathrm{H}_{2} \mathrm{O}_{2}$, which destroys vaginal bacterial components with limited expression of $\mathrm{H}_{2} \mathrm{O}_{2}$ degrading enzymes, including Prevotella and Gardnerella (60, 61). Besides direct inhibition on pathogens, Lactobacillus can occupy possible niches to indirectly protect against pathogen colonization (Figure 2B). For instance, epithelium adhesin facilitates the adhesion of $L$. crispatus to genital mucosa and then additionally inhibits pilus-mediated adhesion of $G$. vaginalis (22).

As discussed above, vaginal Lactobacillus play critical roles in cervicovaginal health, but not all Lactobacillus-dominant CVM benefit the host in the same manner. Lactic acid has D- and Lisomer while the former is mainly produced by $L$. jensenii, $L$. crispatus, L. gasseri and the latter is produced by $L$. iners and a variety of anaerobes (62). Women with $L$. iners- or nonLactobacillus-dominant CVM therefore have a higher ratio of L- and D-lactate, increasing the expression of extracellular matrix metalloproteinase inducer and activating matrix metalloproteinase 8 , which facilitate the entry of HPV to the basal keratinocytes by altering cervical integrity (Figure 2A) (62). Conversely, L. crispatus-dominant CVM can lead to increased cervicovaginal mucus viscosity and promote viral capture (63). Additionally, CVM predominated by L. iners is more instable than CVM with other dominant Lactobacillus species and therefore allows growth of strict anaerobes resulting in transition to non-Lactobacillus-dominant CVM (4, 64). This is consistent with findings that $L$. iners-dominant CVM tends to be identified in women with persistent HPV infection and progression of cervical diseases (Figure 2A) (8, 18, 31, 32, 34). On the contrary, L. crispatus-dominant CVM has the lowest possibility in transition to other CVM types $(4,13,64)$, and is thus positively associated with cervicovaginal health (Figure 2A).

Though many clues exist in microbe-microbe interactions, there are no published reports exploring the mechanism of interaction between CVM and HPV, due to the difficulties of in vitro HPV cultivation. Nevertheless, a growing number of literatures demonstrate unique host immune responses (Figure 2B) (65), which mediate the CVM-HPV interactions in women with HPV infection. Oncoproteins of hrHPV can suppress presentation of hrHPV antigens and impair alarm functions of infected basal keratinocytes where HPV thrive. For example, hrHPV E7 protein can lead to repression of major histocompatibility complex I (MHC I), LMP2 as well as 
TAP1 gene through interaction with MHC I promoter, and E5 protein blocks the transport of MHC I and CD1d to the cell surface, which is crucial for HPV antigen presentation (66-71). Infection of hrHPV also reduce the expression of infected keratinocyte-derived chemokine (C-C motif) ligand 20 (CCL20) $(72,73)$, attracting the migration of LCs which is the only APCs in vaginal epithelium where HPV infection occurs. In addition, hrHPV infection is associated with suppressed LCs activities, including decreased levels of E-cadherin remaining LCs in infected epidermis to capture HPV antigens, and antigenbinding langerin as well as TLR (74-78). Once internalizating HPV antigens, LCs become mature and migrate to lymph nodes via chemokine (C-C-motif) receptor 7 (CCR7) on cell surface. However, prior reports found reduced expression of CCR7 in ICC patients, and identified decreased levels of LCs-membrane antigen-presenting and co-stimulatory molecules in exposure to HPV virus-like particles (VLPs), including CD1a, MHC I, CD40 and $\operatorname{CD} 80 / 86(66,67,75,76,79-81)$.

To the best of our knowledge, no report established in vivo CVM-LCs relationship, which can partly improve the understanding of CVM-HPV interactions. Nevertheless, in vitro experiment found that a $L$ crispatus strain isolated from vagina activated LCs via cell wall-derived PGN, being assessed by elevated expression of TLR (Figure 2B) (25). This is consistent with prior findings that TLR agonists promote LCs activation and the induction of HPV-specific cell-mediated immunity (CMI) (77, 78, 82). Candin, produced by Candida which is inversely associated with HPV infection, can induce proliferation of $\mathrm{T}$ cells to enhance the effect of therapeutic vaccines against $\operatorname{HPV}(44,83,84)$. HerbstKralovetz MM and colleagues also found significant differences of CVM and cervical immune microenvironment between HPVnegative women $(n=18), \mathrm{HPV}$-infected individuals without

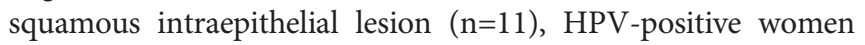
with low $(n=12) /$ high $(n=27)$ intraepithelial lesion, and ICC patients $(n=10)(85-88)$. For instance, inhibitory immune checkpoint protein PD-L1 and LAG-3 were negatively correlated with Lactobacillus abundance in CVM, while TLR2 was in positive relationship with Lactobacillus abundance. Conversely, PD-L1 and LAG-3 positively correlated to dysbiosis-associated Gardnerella, Sneathia, Atopobium and Prevotella. At species level, L. crispatus and $L$. jensenii were in negative relationship with PD-L1, while $L$. gasseri was negatively associated with LAG-3. In addition, a 12month observational study applied the combination of 16S rDNA amplicon sequencing, metagenome, transcriptional profiling and immunological profiling to demonstrate the critical role of cervicovaginal bacteria in modulating cervicovaginal immune responses and the host susceptibility to HIV (23).

\section{APPLICATION OF CVM IN PROMOTING HPV CLEARANCE}

Given the critical roles of CVM in modulating cervical immune responses, it is promising to promote HPV clearance by reconstructing CVM (Figure 3A). Taken vaginal probiotics $L$. crispatus strain CTV-05 for example, a randomized placebo- controlled clinical trial showed that the vaginal colonization with CTV-05 following 28-day treatment inhibited BV-associated Atopobium growth $(89,90)$. Another trial involving 100 participants assessed the efficacy of CTV-05 on preventing urinary tract infection (UTI), indicating the reduction of recurrent UTI when compared to placebo treatment (91). Disrupting biofilm of anaerobes is also an alternative therapy against vaginal dysbiosis, and Marrozzo J. M. et al. found 50-59\% clinical cure rate of BV in 106 participants 9-12 days after treatment (92).

In addition, several in vitro and in vivo studies suggest the promise of prebiotics, which are indigestible carbohydrates, in promoting the growth of probiotics or beneficial commensals in the vagina (Figure 3A) (93-95). For example, fructooligosaccharide (FOS) and gluco-oligosaccharide (GOS) benefited the growth of $L$. crispatus, L. jensenii and L. vaginalis in vitro, while pathogen C. albicans, Escherichia coli and G. vaginalis could not utilize FOS/GOS as energy sources for growth (93). Significant reduction of Nugent scores was also identified in BV patients receiving intravaginal GOS gel immediately following metronidazole treatment (94). Additionally, glucomannan hydrolysates $(\mathrm{GMH})$ also held the potential to promote Lactobacillus spp. colonization, conferring health to the host in C. albicans-infected women (95). To re-establish the CVM completely, two studies in 2019 conducted vaginal microbiota transplantation (VMT) (Figure 3A) (96, 97). A total of 5 women with antibiotic-unresponsive and recurrent BV were included in one study, and 4 out of 5 participants had restoration of Lactobacillus-dominant CVM and long-term remission without any adverse effect at the follow-up of 5-21 months. The other study involving 20 women explained and implemented a screening approach for universal VMT donors.

Besides to CVM-targeted interventions, CVM-derived products hold the promise as immune modulators, such as adjuvants of therapeutic vaccines (Figure 3B). Jie Song and co-workers demonstrated that PGN produced by a vaginal L. crispatus strain enhanced the expression of cell-membrane TLR2 and TLR6 to activate LCs (25), which play a pivotal role in capturing and presenting HPV antigens. The products of specific bacterial components have the potential to be effective adjuvants as a series of clinical trials demonstrated enhanced efficacy of therapeutic vaccines adjuvanted with TLR agonists which could be served by bacterial products (98-101). Furthermore, bacterial vectors are increasingly explored as alternative live vectors due to their potential as "natural" adjuvants, which attributed to the wide range of pathogen-associated molecular pattern molecules and damage-associated molecular pattern molecules (102-106). Additionally, candin produced by common vaginal fungal pathobiont Candida could be utilized as adjuvant for therapeutic vaccine, which partly explain the protection of vaginal Candida against HPV infection $(44,83,84)$.

\section{CONCLUSION}

CVM appears to play a crucial role in HPV acquisition and persistence as well as subsequent development of squamous 

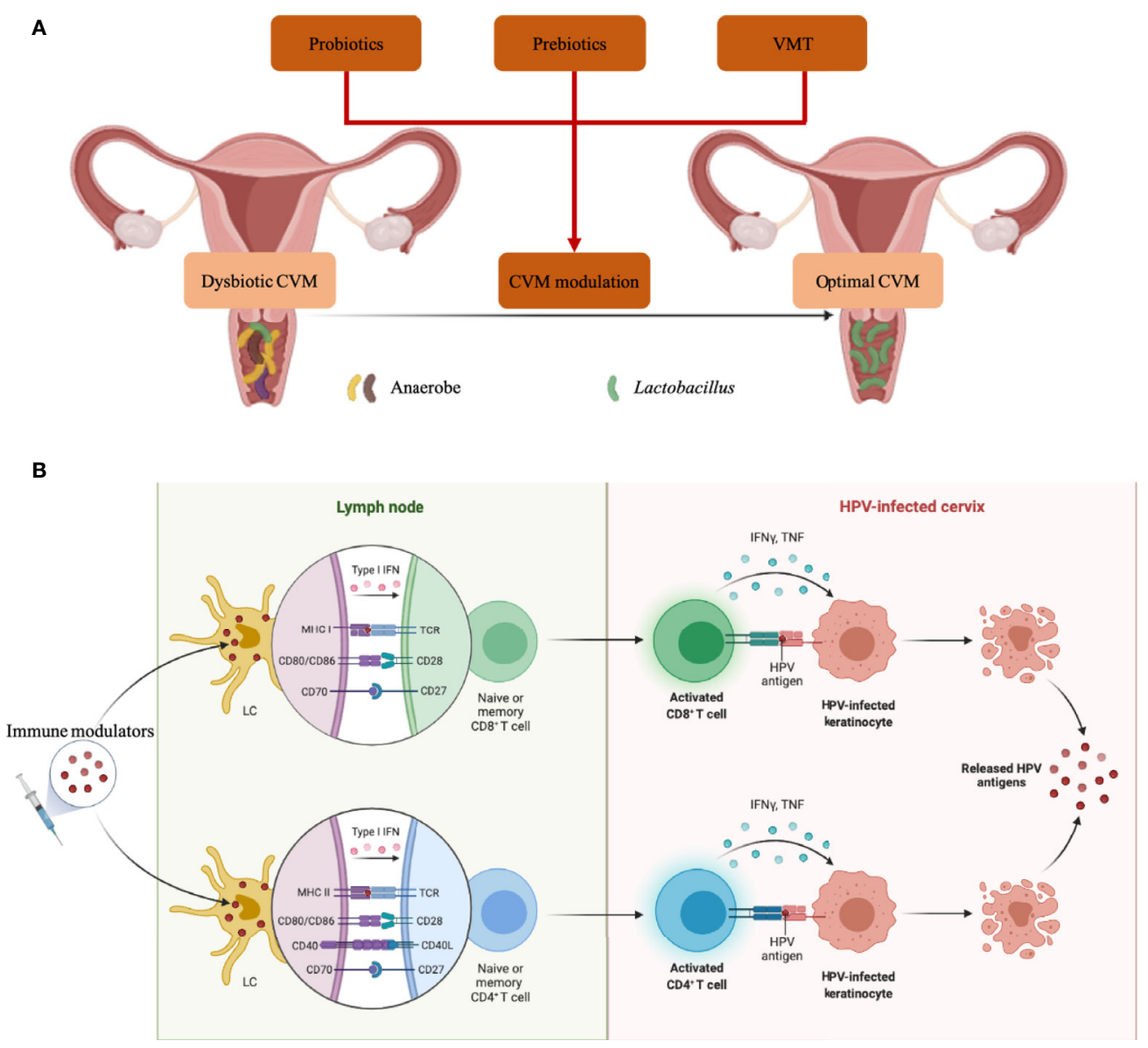

FIGURE 3 | CVM-modulated and -derived products in eliminating HPV infection. (A) Strategies to modulate dysbiotic CVM were characterized by anaerobe overgrowth. (B) Molecules produced by specific microbial strains in CVM can activate LCs in the cervical epithelium, then promote T cell priming and eliminate HPVinfected cells. Icons in BioRender.com were utilized to prepare this figure.

intraepithelial lesion. Cross-sectional nature of most studies makes it difficult to derive a causal link between CVM and HPV infection or clearance. In addition, many prior reports described CVM in relatively small cohorts, which analysis results could be compounded by various factors, such as smoking and sex activities. Prospective cohort study will be needed in the future to prove that CVM could prevent HPV infection and promote HPV clearance. This information will determine the promise of CVM interventions as novel therapies, with the advantage of low-cost feasibility in developing countries. Nevertheless, it is imperative to find the most protective strains before developing CVM-targeted probiotics or prebiotics, for which the efficacy can be impacted by pre-existed CVM. For example, L. crispatus-dominated CVM confers high colonization resistance to other microbes and even probiotic L. crispatus strain, while pre-colonization of the vagina with endogenous $L$. iners allows growth of anaerobes. Therefore, CVM structure should be taken into consideration when it comes to assess the efficacy of specific probiotics and prebiotics. However, 16S rDNA amplicon sequencing that most studies applied has limitations in conducting strain-level analysis and microbemicrobe/host interactions of CVM, necessitating the utilization of multi-omics in analyzing "key microbial strains". Then mechanistic studies of these strains should be conducted to further the utilization of "key microbial strains" as immune modulators in prevention and clearance of HPV infection. Given the importance of cervical epithelial LCs in presenting HPV antigens to induce HPV-specific CMI, it will be an effective mediator of therapeutic vaccine immunity. As discussed above, specific microbial strains in CVM hold the potential to activate HPV-suppressed LCs, suggesting the promise of microbial products as robust activator of immunity against HPV or adjuvants in therapeutic vaccines. In the future, the combination of culture-independent and -dependent techniques should be applied to screen promising microbial strains and products which functions can be assessed in cell lines or animal models. Lastly, though VMT can modify the whole cervicovaginal microenvironment, randomized, placebocontrolled studies for large cohorts are required to determine the clinical efficacy as well as long-term benefits. 


\section{AUTHOR CONTRIBUTIONS}

WD and RW made substantial contributions to the design and writing of this manuscript. HD and SL contributed to the discussion and conception of the work. All authors contributed to the article and approved the submitted version.

\section{REFERENCES}

1. Bray F, Ferlay J, Soerjomataram I, Siegel RL, Torre LA, Jemal A. Global Cancer Statistics 2018: GLOBOCAN Estimates of Incidence and Mortality Worldwide for 36 Cancers in 185 Countries. CA Cancer J Clin (2018) 68 (6):394-424. doi: 10.3322/caac.21492

2. Hildesheim A, Herrero R, Wacholder S, Rodriguez AC, Solomon D, Bratti MC, et al. Effect of Human Papillomavirus 16/18 L1 Viruslike Particle Vaccine Among Young Women With Preexisting Infection: A Randomized Trial. JAMA (2007) 298(7):743-53. doi: 10.1001/jama.298.7.743

3. Group FIS. Quadrivalent Vaccine Against Human Papillomavirus to Prevent High-Grade Cervical Lesions. N Engl J Med (2007) 356(19):191527. doi: 10.1056/NEJMoa061741

4. Mitra A, MacIntyre DA, Ntritsos G, Smith A, Tsilidis KK, Marchesi JR, et al. The Vaginal Microbiota Associates With the Regression of Untreated Cervical Intraepithelial Neoplasia 2 Lesions. Nat Commun (2020) 11 (1):1999. doi: 10.1038/s41467-020-15856-y

5. Usyk M, Zolnik CP, Castle PE, Porras C, Herrero R, Gradissimo A, et al. Cervicovaginal Microbiome and Natural History of HPV in a Longitudinal Study. PloS Pathog (2020) 16(3):e1008376. doi: 10.1371/journal. ppat.1008376

6. Berggrund M, Gustavsson I, Aarnio R, Lindberg JH, Sanner K, Wikstrom I, et al. Temporal Changes in the Vaginal Microbiota in Self-Samples and its Association With Persistent HPV16 Infection and CIN2. Virol J (2020) 17 (1):147. doi: 10.1186/s12985-020-01420-Z

7. Brotman RM, Shardell MD, Gajer P, Tracy JK, Zenilman JM, Ravel J, et al. Interplay Between the Temporal Dynamics of the Vaginal Microbiota and Human Papillomavirus Detection. J Infect Dis (2014) 210(11):1723-33. doi: 10.1093/infdis/jiu330

8. Di Paola M, Sani C, Clemente AM, Iossa A, Perissi E, Castronovo G, et al. Characterization of Cervico-Vaginal Microbiota in Women Developing Persistent High-Risk Human Papillomavirus Infection. Sci Rep (2017) 7 (1):10200. doi: 10.1038/s41598-017-09842-6

9. Al-Nasiry S, Ambrosino E, Schlaepfer M, Morre SA, Wieten L, Voncken JW, et al. The Interplay Between Reproductive Tract Microbiota and Immunological System in Human Reproduction. Front Immunol (2020) 11:378. doi: 10.3389/fimmu.2020.00378

10. Chen C, Song X, Wei W, Zhong H, Dai J, Lan Z, et al. The Microbiota Continuum Along the Female Reproductive Tract and Its Relation to Uterine-Related Diseases. Nat Commun (2017) 8(1):875. doi: 10.1038/ s41467-017-00901-0

11. Ma B, France MT, Crabtree J, Holm JB, Humphrys MS, Brotman RM, et al. A Comprehensive Non-Redundant Gene Catalog Reveals Extensive WithinCommunity Intraspecies Diversity in the Human Vagina. Nat Commun (2020) 11(1):940. doi: 10.1038/s41467-020-14677-3

12. Ravel J, Gajer P, Abdo Z, Schneider GM, Koenig SS, McCulle SL, et al. Vaginal Microbiome of Reproductive-Age Women. Proc Natl Acad Sci U S A (2011) 108(Suppl 1):4680-7. doi: 10.1073/pnas.1002611107

13. Gajer P, Brotman RM, Bai G, Sakamoto J, Schutte UM, Zhong X, et al. Temporal Dynamics of the Human Vaginal Microbiota. Sci Transl Med (2012) 4(132):132ra52. doi: 10.1126/scitranslmed.3003605

14. Chaban B, Links MG, Jayaprakash TP, Wagner EC, Bourque DK, Lohn Z, et al. Characterization of the Vaginal Microbiota of Healthy Canadian Women Through the Menstrual Cycle. Microbiome (2014) 2:23. doi: 10.1186/2049-2618-2-23

15. Kovachev S. Defence Factors of Vaginal Lactobacilli. Crit Rev Microbiol (2018) 44(1):31-9. doi: 10.1080/1040841X.2017.1306688

16. Cheng L, Norenhag J, Hu YOO, Brusselaers N, Fransson E, Ahrlund-Richter A, et al. Vaginal Microbiota and Human Papillomavirus Infection Among

\section{FUNDING}

This work was supported by Shenzhen High-level Hospital Construction Fund (YBH2019-260), Shenzhen Key Medical Discipline Construction Fund (No. SZXK027) and Sanming Project of Medicine in Shenzhen (No. SZSM202011016).

Young Swedish Women. NPJ Biofilms Microbiomes (2020) 6(1):39. doi: 10.1038/s41522-020-00146-8

17. Zhou Y, Wang L, Pei F, Ji M, Zhang F, Sun Y, et al. Patients With Lr-Hpv Infection Have a Distinct Vaginal Microbiota in Comparison With Healthy Controls. Front Cell Infect Microbiol (2019) 9:294. doi: 10.3389/fcimb. 2019.00294

18. Oh HY, Kim BS, Seo SS, Kong JS, Lee JK, Park SY, et al. The Association of Uterine Cervical Microbiota With an Increased Risk for Cervical Intraepithelial Neoplasia in Korea. Clin Microbiol Infect (2015) 21(7):674 e1-9. doi: 10.1016/j.cmi.2015.02.026

19. Mitra A, MacIntyre DA, Lee YS, Smith A, Marchesi JR, Lehne B, et al Cervical Intraepithelial Neoplasia Disease Progression Is Associated With Increased Vaginal Microbiome Diversity. Sci Rep (2015) 5:16865. doi: $10.1038 /$ srep 16865

20. Borgogna JC, Shardell MD, Santori EK, Nelson TM, Rath JM, Glover ED, et al. The Vaginal Metabolome and Microbiota of Cervical HPV-Positive and HPV-Negative Women: A Cross-Sectional Analysis. BJOG (2020) 127 (2):182-92. doi: 10.1111/1471-0528.15981

21. Lee JE, Lee S, Lee H, Song YM, Lee K, Han MJ, et al. Association of the Vaginal Microbiota With Human Papillomavirus Infection in a Korean Twin Cohort. PloS One (2013) 8(5):e63514. doi: 10.1371/journal.pone.0063514

22. Ojala T, Kankainen M, Castro J, Cerca N, Edelman S, Westerlund-Wikstrom B, et al. Comparative Genomics of Lactobacillus Crispatus Suggests Novel Mechanisms for the Competitive Exclusion of Gardnerella Vaginalis. BMC Genomics (2014) 15:1070. doi: 10.1186/1471-2164-15-1070

23. Anahtar MN, Byrne EH, Doherty KE, Bowman BA, Yamamoto HS, Soumillon M, et al. Cervicovaginal Bacteria Are a Major Modulator of Host Inflammatory Responses in the Female Genital Tract. Immunity (2015) 42(5):965-76. doi: 10.1016/j.immuni.2015.04.019

24. Doerflinger SY, Throop AL, Herbst-Kralovetz MM. Bacteria in the Vaginal Microbiome Alter the Innate Immune Response and Barrier Properties of the Human Vaginal Epithelia in a Species-Specific Manner. J Infect Dis (2014) 209(12):1989-99. doi: 10.1093/infdis/jiu004

25. Song J, Lang F, Zhao N, Guo Y, Zhang H. Vaginal Lactobacilli Induce Differentiation of Monocytic Precursors Toward Langerhans-Like Cells: In Vitro Evidence. Front Immunol (2018) 9:2437. doi: 10.3389/fimmu.2018.02437

26. Shannon B, Yi TJ, Perusini S, Gajer P, Ma B, Humphrys MS, et al. Association of HPV Infection and Clearance With Cervicovaginal Immunology and the Vaginal Microbiota. Mucosal Immunol (2017) 10 (5):1310-9. doi: 10.1038/mi.2016.129

27. Kindt N, Descamps G, Seminerio I, Bellier J, Lechien JR, Pottier C, et al. Langerhans Cell Number Is a Strong and Independent Prognostic Factor for Head and Neck Squamous Cell Carcinomas. Oral Oncol (2016) 62:1-10. doi: 10.1016/j.oraloncology.2016.08.016

28. Miyagi J, Kinjo T, Tsuhako K, Higa M, Iwamasa T, Kamada Y, et al. Extremely High Langerhans Cell Infiltration Contributes to the Favourable Prognosis of HPV-Infected Squamous Cell Carcinoma and Adenocarcinoma of the Lung. Histopathology (2001) 38(4):355-67. doi: 10.1046/j.13652559.2001.01067.x

29. Chao XP, Sun TT, Wang S, Fan QB, Shi HH, Zhu L, et al. Correlation Between the Diversity of Vaginal Microbiota and the Risk of High-Risk Human Papillomavirus Infection. Int J Gynecol Cancer (2019) 29(1):28-34. doi: 10.1136/ijgc-2018-000032

30. Gao W, Weng J, Gao Y, Chen X. Comparison of the Vaginal Microbiota Diversity of Women With and Without Human Papillomavirus Infection: A Cross-Sectional Study. BMC Infect Dis (2013) 13:271. doi: 10.1186/14712334-13-271

31. So KA, Yang EJ, Kim NR, Hong SR, Lee JH, Hwang CS, et al. Changes of Vaginal Microbiota During Cervical Carcinogenesis in Women With 
Human Papillomavirus Infection. PloS One (2020) 15(9):e0238705. doi: 10.1371/journal.pone.0238705

32. Zhang C, Liu Y, Gao W, Pan Y, Gao Y, Shen J, et al. The Direct and Indirect Association of Cervical Microbiota With the Risk of Cervical Intraepithelial Neoplasia. Cancer Med (2018) 7(5):2172-9. doi: 10.1002/cam4.1471

33. Liang $Y$, Chen M, Qin L, Wan B, Wang H. A Meta-Analysis of the Relationship Between Vaginal Microecology, Human Papillomavirus Infection and Cervical Intraepithelial Neoplasia. Infect Agent Cancer (2019) 14:29. doi: 10.1186/s13027-019-0243-8

34. Seo SS, Oh HY, Lee JK, Kong JS, Lee DO, Kim MK. Combined Effect of Diet and Cervical Microbiome on the Risk of Cervical Intraepithelial Neoplasia. Clin Nutr (2016) 35(6):1434-41. doi: 10.1016/j.clnu.2016.03.019

35. Norenhag J, Du J, Olovsson M, Verstraelen H, Engstrand L, Brusselaers N. The Vaginal Microbiota, Human Papillomavirus and Cervical Dysplasia: A Systematic Review and Network Meta-Analysis. BJOG (2020) 127(2):17180. doi: 10.1111/1471-0528.15854

36. Wang H, Ma Y, Li R, Chen X, Wan L, Zhao W. Associations of Cervicovaginal Lactobacilli With High-Risk Human Papillomavirus Infection, Cervical Intraepithelial Neoplasia, and Cancer: A Systematic Review and Meta-Analysis. J Infect Dis (2019) 220(8):1243-54. doi: 10.1093/infdis/jiz325

37. Tango CN, Seo SS, Kwon M, Lee DO, Chang HK, Kim MK. Taxonomic and Functional Differences in Cervical Microbiome Associated With Cervical Cancer Development. Sci Rep (2020) 10(1):9720. doi: 10.1038/s41598-02066607-4

38. Kwon M, Seo SS, Kim MK, Lee DO, Lim MC. Compositional and Functional Differences Between Microbiota and Cervical Carcinogenesis as Identified by Shotgun Metagenomic Sequencing. Cancers (Basel) (2019) 11(3):309. doi: 10.3390/cancers11030309

39. Donmez HG, Sahal G, Akgor U, Cagan M, Ozgul N, Beksac MS. The Relationship Between the Presence of HPV Infection and Biofilm Formation in Cervicovaginal Smears. Infection (2020) 48(5):735-40. doi: 10.1007/ s15010-020-01478-5

40. Dareng EO, Ma B, Adebamowo SN, Famooto A, Ravel J, Pharoah PP, et al. Vaginal Microbiota Diversity and Paucity of Lactobacillus Species Are Associated With Persistent hrHPV Infection in HIV Negative But Not in HIV Positive Women. Sci Rep (2020) 10(1):19095. doi: 10.1038/s41598-020-76003-7

41. Reimers LL, Mehta SD, Massad LS, Burk RD, Xie X, Ravel J, et al. The Cervicovaginal Microbiota and Its Associations With Human Papillomavirus Detection in HIV-Infected and HIV-Uninfected Women. J Infect Dis (2016) 214 (9):1361-9. doi: 10.1093/infdis/jiw374

42. Tamarelle J, Thiebaut ACM, de Barbeyrac B, Bebear C, Ravel J, DelarocqueAstagneau E. The Vaginal Microbiota and its Association With Human Papillomavirus, Chlamydia Trachomatis, Neisseria Gonorrhoeae and Mycoplasma Genitalium Infections: A Systematic Review and MetaAnalysis. Clin Microbiol Infect (2019) 25(1):35-47. doi: 10.1016/ j.cmi.2018.04.019

43. Brusselaers N, Shrestha S, van de Wijgert J, Verstraelen H. Vaginal Dysbiosis and the Risk of Human Papillomavirus and Cervical Cancer: Systematic Review and Meta-Analysis. Am J Obstet Gynecol (2019) 221(1):9-18.e8. doi: 10.1016/j.ajog.2018.12.011

44. Engberts MK, Verbruggen BS, Boon ME, van Haaften M, Heintz AP. Candida and Dysbacteriosis: A Cytologic, Population-Based Study of 100,605 Asymptomatic Women Concerning Cervical Carcinogenesis. Cancer (2007) 111(5):269-74. doi: 10.1002/cncr.22947

45. Briselden AM, Moncla BJ, Stevens CE, Hillier SL. Sialidases (Neuraminidases) in Bacterial Vaginosis and Bacterial VaginosisAssociated Microflora. J Clin Microbiol (1992) 30(3):663-6. doi: 10.1128/ JCM.30.3.663-666.1992

46. Holmes KK, Chen KC, Lipinski CM, Eschenbach DA. Vaginal Redox Potential in Bacterial Vaginosis (Nonspecific Vaginitis). J Infect Dis (1985) 152(2):379-82. doi: 10.1093/infdis/152.2.379

47. Anderson BL, Cu-Uvin S, Raker CA, Fitzsimmons C, Hillier SL. Subtle Perturbations of Genital Microflora Alter Mucosal Immunity Among LowRisk Pregnant Women. Acta Obstet Gynecol Scand (2011) 90(5):510-5. doi: 10.1111/j.1600-0412.2011.01082.x

48. Hedges SR, Barrientes F, Desmond RA, Schwebke JR. Local and Systemic Cytokine Levels in Relation to Changes in Vaginal Flora. J Infect Dis (2006) 193(4):556-62. doi: 10.1086/499824
49. Uren A, Fallen S, Yuan H, Usubutun A, Kucukali T, Schlegel R, et al. Activation of the Canonical Wnt Pathway During Genital Keratinocyte Transformation: A Model for Cervical Cancer Progression. Cancer Res (2005) 65(14):6199-206. doi: 10.1158/0008-5472.CAN-05-0455

50. Cheriyan VT, Krishna SM, Kumar A, Jayaprakash PG, Balaram P. Signaling Defects and Functional Impairment in T-Cells From Cervical Cancer Patients. Cancer Biother Radiopharm (2009) 24(6):667-73. doi: 10.1089/ cbr.2009.0660

51. Gur C, Ibrahim Y, Isaacson B, Yamin R, Abed J, Gamliel M, et al. Binding of the Fap2 Protein of Fusobacterium Nucleatum to Human Inhibitory Receptor TIGIT Protects Tumors From Immune Cell Attack. Immunity (2015) 42(2):344-55. doi: 10.1016/j.immuni.2015.01.010

52. Mastromarino P, Di Pietro M, Schiavoni G, Nardis C, Gentile M, Sessa R. Effects of Vaginal Lactobacilli in Chlamydia Trachomatis Infection. Int $J$ Med Microbiol (2014) 304(5-6):654-61. doi: 10.1016/j.ijmm.2014.04.006

53. Breshears LM, Edwards VL, Ravel J, Peterson ML. Lactobacillus Crispatus Inhibits Growth of Gardnerella Vaginalis and Neisseria Gonorrhoeae on a Porcine Vaginal Mucosa Model. BMC Microbiol (2015) 15:276. doi: 10.1186/ s12866-015-0608-0

54. Gong Z, Luna Y, Yu P, Fan H. Lactobacilli Inactivate Chlamydia Trachomatis Through Lactic Acid But Not H2O2. PloS One (2014) 9(9): e107758. doi: 10.1371/journal.pone.0107758

55. Graver MA, Wade JJ. The Role of Acidification in the Inhibition of Neisseria Gonorrhoeae by Vaginal Lactobacilli During Anaerobic Growth. Ann Clin Microbiol Antimicrob (2011) 10:8. doi: 10.1186/1476-0711-10-8

56. Stoyancheva G, Marzotto M, Dellaglio F, Torriani S. Bacteriocin Production and Gene Sequencing Analysis From Vaginal Lactobacillus Strains. Arch Microbiol (2014) 196(9):645-53. doi: 10.1007/s00203-014-1003-1

57. Dover SE, Aroutcheva AA, Faro S, Chikindas ML. Natural Antimicrobials and Their Role in Vaginal Health: A Short Review. Int J Probiotics Prebiotics (2008) 3(4):219-30.

58. Reid G, Heinemann C, Velraeds M, van der Mei HC, Busscher HJ. Biosurfactants Produced by Lactobacillus. Methods Enzymol (1999) 310:426-33. doi: 10.1016/s0076-6879(99)10033-8

59. Zarate G, Nader-Macias ME. Influence of Probiotic Vaginal Lactobacilli on In Vitro Adhesion of Urogenital Pathogens to Vaginal Epithelial Cells. Lett Appl Microbiol (2006) 43(2):174-80. doi: 10.1111/j.1472-765X.2006.01934.x

60. Strus M, Brzychczy-Wloch M, Gosiewski T, Kochan P, Heczko PB. The In Vitro Effect of Hydrogen Peroxide on Vaginal Microbial Communities. FEMS Immunol Med Microbiol (2006) 48(1):56-63. doi: 10.1111/j.1574695X.2006.00120. $\mathrm{x}$

61. Vallor AC, Antonio MA, Hawes SE, Hillier SL. Factors Associated With Acquisition of, or Persistent Colonization by, Vaginal Lactobacilli: Role of Hydrogen Peroxide Production. J Infect Dis (2001) 184(11):1431-6. doi: $10.1086 / 324445$

62. Witkin SS, Mendes-Soares H, Linhares IM, Jayaram A, Ledger WJ, Forney LJ. Influence of Vaginal Bacteria and D- and L-Lactic Acid Isomers on Vaginal Extracellular Matrix Metalloproteinase Inducer: Implications for Protection Against Upper Genital Tract Infections. mBio (2013) 4(4): e00460-13. doi: 10.1128/mBio.00460-13

63. Nunn KL, Wang YY, Harit D, Humphrys MS, Ma B, Cone R, et al. Enhanced Trapping of HIV-1 by Human Cervicovaginal Mucus Is Associated With Lactobacillus crispatus-Dominant Microbiota. mBio (2015) 6(5):e01084-15. doi: 10.1128/mBio.01084-15

64. Romero R, Hassan SS, Gajer P, Tarca AL, Fadrosh DW, Nikita L, et al. The Composition and Stability of the Vaginal Microbiota of Normal Pregnant Women Is Different From That of non-Pregnant Women. Microbiome (2014) 2(1):4. doi: 10.1186/2049-2618-2-4

65. Zhou C, Tuong ZK, Frazer IH. Papillomavirus Immune Evasion Strategies Target the Infected Cell and the Local Immune System. Front Oncol (2019) 9:682. doi: 10.3389/fonc.2019.00682

66. Georgopoulos NT, Proffitt JL, Blair GE. Transcriptional Regulation of the Major Histocompatibility Complex (MHC) Class I Heavy Chain, TAP1 and LMP2 Genes by the Human Papillomavirus (HPV) Type 6b, 16 and 18 E7 Oncoproteins. Oncogene (2000) 19(42):4930-5. doi: 10.1038/sj.onc.1203860

67. Heller C, Weisser T, Mueller-Schickert A, Rufer E, Hoh A, Leonhardt RM, et al. Identification of Key Amino Acid Residues That Determine the Ability of High Risk HPV16-E7 to Dysregulate Major Histocompatibility Complex 
Class I Expression. J Biol Chem (2011) 286(13):10983-97. doi: 10.1074/ jbc.M110.199190

68. Zhou F, Leggatt GR, Frazer IH. Human Papillomavirus 16 E7 Protein Inhibits Interferon-Gamma-Mediated Enhancement of Keratinocyte Antigen Processing and T-Cell Lysis. FEBS J (2011) 278(6):955-63. doi: 10.1111/j.1742-4658.2011.08011.x

69. Cortese MS, Ashrafi GH, Campo MS. All 4 Di-Leucine Motifs in the First Hydrophobic Domain of the E5 Oncoprotein of Human Papillomavirus Type 16 Are Essential for Surface MHC Class I Downregulation Activity and E5 Endomembrane Localization. Int J Cancer (2010) 126(7):1675-82. doi: $10.1002 / \mathrm{ijc} .25004$

70. Gruener M, Bravo IG, Momburg F, Alonso A, Tomakidi P. The E5 Protein of the Human Papillomavirus Type 16 Down-Regulates HLA-I Surface Expression in Calnexin-Expressing But Not in Calnexin-Deficient Cells. Virol J (2007) 4:116. doi: 10.1186/1743-422X-4-116

71. Miura S, Kawana K, Schust DJ, Fujii T, Yokoyama T, Iwasawa Y, et al. CD1d, a Sentinel Molecule Bridging Innate and Adaptive Immunity, Is Downregulated by the Human Papillomavirus (HPV) E5 Protein: A Possible Mechanism for Immune Evasion by HPV. J Virol (2010) 84 (22):11614-23. doi: 10.1128/JVI.01053-10

72. Sperling T, Oldak M, Walch-Ruckheim B, Wickenhauser C, Doorbar J, Pfister H, et al. Human Papillomavirus Type 8 Interferes With a Novel C/ EBPbeta-Mediated Mechanism of Keratinocyte CCL20 Chemokine Expression and Langerhans Cell Migration. PloS Pathog (2012) 8(7): e1002833. doi: 10.1371/journal.ppat.1002833

73. Guess JC, McCance DJ. Decreased Migration of Langerhans Precursor-Like Cells in Response to Human Keratinocytes Expressing Human Papillomavirus Type 16 E6/E7 Is Related to Reduced Macrophage Inflammatory protein-3alpha Production. J Virol (2005) 79(23):14852-62. doi: 10.1128/JVI.79.23.14852-14862.2005

74. Laurson J, Khan S, Chung R, Cross K, Raj K. Epigenetic Repression of ECadherin by Human Papillomavirus 16 E7 Protein. Carcinogenesis (2010) 31 (5):918-26. doi: 10.1093/carcin/bgq027

75. Hubert P, Caberg JH, Gilles C, Bousarghin L, Franzen-Detrooz E, Boniver J, et al. E-Cadherin-Dependent Adhesion of Dendritic and Langerhans Cells to Keratinocytes Is Defective in Cervical Human Papillomavirus-Associated (Pre)Neoplastic Lesions. J Pathol (2005) 206(3):346-55. doi: 10.1002/ path. 1771

76. Connor JP, Ferrer K, Kane JP, Goldberg JM. Evaluation of Langerhans' Cells in the Cervical Epithelium of Women With Cervical Intraepithelial Neoplasia. Gynecol Oncol (1999) 75(1):130-5. doi: 10.1006/gyno.1999.5559

77. Fahey LM, Raff AB, Da Silva DM, Kast WM. Reversal of Human Papillomavirus-Specific T Cell Immune Suppression Through TLR Agonist Treatment of Langerhans Cells Exposed to Human Papillomavirus Type 16. J Immunol (2009) 182(5):2919-28. doi: 10.4049/ jimmunol.0803645

78. Kumar MM, Adurthi S, Ramachandran S, Mukherjee G, Joy O, Krishnamurthy H, et al. Toll-Like Receptors 7, 8, and 9 Expression and Function in Primary Human Cervical Cancer Langerhans Cells: Evidence of Anergy. Int J Gynecol Cancer (2013) 23(1):184-92. doi: 10.1097/ IGC.0b013e31827a2003

79. Pahne-Zeppenfeld J, Schroer N, Walch-Ruckheim B, Oldak M, Gorter A, Hegde S, et al. Cervical Cancer Cell-Derived Interleukin-6 Impairs CCR7Dependent Migration of MMP-9-Expressing Dendritic Cells. Int J Cancer (2014) 134(9):2061-73. doi: 10.1002/ijc.28549

80. Da Silva DM, Movius CA, Raff AB, Brand HE, Skeate JG, Wong MK, et al. Suppression of Langerhans Cell Activation Is Conserved Amongst Human Papillomavirus Alpha and Beta Genotypes, But Not a Micro Genotype. Virology (2014) 452-453:279-86. doi: 10.1016/j.virol.2014.01.031

81. Fausch SC, Da Silva DM, Rudolf MP, Kast WM. Human Papillomavirus Virus-Like Particles do Not Activate Langerhans Cells: A Possible Immune Escape Mechanism Used by Human Papillomaviruses. J Immunol (2002) 169(6):3242-9. doi: 10.4049/jimmunol.169.6.3242

82. Da Silva DM, Woodham AW, Skeate JG, Rijkee LK, Taylor JR, Brand HE, et al. Langerhans Cells From Women With Cervical Precancerous Lesions Become Functionally Responsive Against Human Papillomavirus After Activation With Stabilized Poly-I:C. Clin Immunol (2015) 161(2):197-208. doi: 10.1016/j.clim.2015.09.003
83. Coleman HN, Greenfield WW, Stratton SL, Vaughn R, Kieber A, MoermanHerzog AM, et al. Human Papillomavirus Type 16 Viral Load Is Decreased Following a Therapeutic Vaccination. Cancer Immunol Immunother (2016) 65(5):563-73. doi: 10.1007/s00262-016-1821-x

84. Kim KH, Horn TD, Pharis J, Kincannon J, Jones R, O'Bryan K, et al. Phase 1 Clinical Trial of Intralesional Injection of Candida Antigen for the Treatment of Warts. Arch Dermatol (2010) 146(12):1431-3. doi: 10.1001/ archdermatol.2010.350

85. Laniewski P, Barnes D, Goulder A, Cui H, Roe DJ, Chase DM, et al. Linking Cervicovaginal Immune Signatures, HPV and Microbiota Composition in Cervical Carcinogenesis in Non-Hispanic and Hispanic Women. Sci Rep (2018) 8(1):7593. doi: 10.1038/s41598-018-25879-7

86. Ilhan ZE, Laniewski P, Thomas N, Roe DJ, Chase DM, Herbst-Kralovetz MM. Deciphering the Complex Interplay Between Microbiota, HPV, Inflammation and Cancer Through Cervicovaginal Metabolic Profiling. EBioMedicine (2019) 44:675-90. doi: 10.1016/j.ebiom.2019.04.028

87. Laniewski P, Cui H, Roe DJ, Barnes D, Goulder A, Monk BJ, et al. Features of the Cervicovaginal Microenvironment Drive Cancer Biomarker Signatures in Patients Across Cervical Carcinogenesis. Sci Rep (2019) 9(1):7333. doi: 10.1038/s41598-019-43849-5

88. Laniewski P, Cui H, Roe DJ, Chase DM, Herbst-Kralovetz MM. Vaginal Microbiota, Genital Inflammation, and Neoplasia Impact Immune Checkpoint Protein Profiles in the Cervicovaginal Microenvironment. NPJ Precis Oncol (2020) 4:22. doi: 10.1038/s41698-020-0126-x

89. Ngugi BM, Hemmerling A, Bukusi EA, Kikuvi G, Gikunju J, Shiboski S, et al. Effects of Bacterial Vaginosis-Associated Bacteria and Sexual Intercourse on Vaginal Colonization With the Probiotic Lactobacillus Crispatus CTV-05. Sex Transm Dis (2011) 38(11):1020-7. doi: 10.1097/OLQ.0b013e3182267ac4

90. Hemmerling A, Harrison W, Schroeder A, Park J, Korn A, Shiboski S, et al. Phase 2a Study Assessing Colonization Efficiency, Safety, and Acceptability of Lactobacillus Crispatus CTV-05 in Women With Bacterial Vaginosis. Sex Transm Dis (2010) 37(12):745-50. doi: 10.1097/OLQ.0b013e3181e50026

91. Stapleton AE, Au-Yeung M, Hooton TM, Fredricks DN, Roberts PL, Czaja CA, et al. Randomized, Placebo-Controlled Phase 2 Trial of a Lactobacillus Crispatus Probiotic Given Intravaginally for Prevention of Recurrent Urinary Tract Infection. Clin Infect Dis (2011) 52(10):1212-7. doi: 10.1093/cid/cir183

92. Marrazzo JM, Dombrowski JC, Wierzbicki MR, Perlowski C, Pontius A, Dithmer D, et al. Safety and Efficacy of a Novel Vaginal Anti-infective, TOL463, in the Treatment of Bacterial Vaginosis and Vulvovaginal Candidiasis: A Randomized, Single-Blind, Phase 2, Controlled Trial. Clin Infect Dis (2019) 68(5):803-9. doi: 10.1093/cid/ciy554

93. Rousseau V, Lepargneur JP, Roques C, Remaud-Simeon M, Paul F. Prebiotic Effects of Oligosaccharides on Selected Vaginal Lactobacilli and Pathogenic Microorganisms. Anaerobe (2005) 11(3):145-53. doi: 10.1016/j.anaerobe. 2004.12.002

94. Coste I, Judlin P, Lepargneur JP, Bou-Antoun S. Safety and Efficacy of an Intravaginal Prebiotic Gel in the Prevention of Recurrent Bacterial Vaginosis: A Randomized Double-Blind Study. Obstet Gynecol Int (2012) 2012:147867. doi: 10.1155/2012/147867

95. Tester R, Al-Ghazzewi F, Shen N, Chen Z, Chen F, Yang J, et al. The Use of Konjac Glucomannan Hydrolysates to Recover Healthy Microbiota in Infected Vaginas Treated With an Antifungal Agent. Benef Microbes (2012) 3(1):61-6. doi: 10.3920/BM2011.0021

96. Lev-Sagie A, Goldman-Wohl D, Cohen Y, Dori-Bachash M, Leshem A, Mor U, et al. Vaginal Microbiome Transplantation in Women With Intractable Bacterial Vaginosis. Nat Med (2019) 25(10):1500-4. doi: 10.1038/s41591019-0600-6

97. DeLong K, Bensouda S, Zulfiqar F, Zierden HC, Hoang TM, Abraham AG, et al. Conceptual Design of a Universal Donor Screening Approach for Vaginal Microbiota Transplant. Front Cell Infect Microbiol (2019) 9:306. doi: $10.3389 /$ fcimb.2019.00306

98. Da Silva DM, Skeate JG, Chavez-Juan E, Luhen KP, Wu JM, Wu CM, et al. Therapeutic Efficacy of a Human Papillomavirus Type 16 E7 Bacterial Exotoxin Fusion Protein Adjuvanted With CpG or GPI-0100 in a Preclinical Mouse Model for HPV-Associated Disease. Vaccine (2019) 37 (22):2915-24. doi: 10.1016/j.vaccine.2019.04.043

99. Maynard SK, Marshall JD, MacGill RS, Yu L, Cann JA, Cheng LI, et al. Vaccination With Synthetic Long Peptide Formulated With CpG in an Oil- 
in-Water Emulsion Induces Robust E7-Specific CD8 T Cell Responses and TC-1 Tumor Eradication. BMC Cancer (2019) 19(1):540. doi: 10.1186/ s12885-019-5725-y

100. Gandhapudi SK, Ward M, Bush JPC, Bedu-Addo F, Conn G, Woodward JG. Antigen Priming With Enantiospecific Cationic Lipid Nanoparticles Induces Potent Antitumor CTL Responses Through Novel Induction of a Type I IFN Response. J Immunol (2019) 202(12):3524-36. doi: 10.4049/jimmunol.1801634

101. Daayana S, Elkord E, Winters U, Pawlita M, Roden R, Stern PL, et al. Phase II Trial of Imiquimod and HPV Therapeutic Vaccination in Patients With Vulval Intraepithelial Neoplasia. Br J Cancer (2010) 102(7):1129-36. doi: 10.1038/sj.bjc.6605611

102. Ribelles P, Benbouziane B, Langella P, Suarez JE, Bermudez-Humaran LG. Protection Against Human Papillomavirus Type 16-Induced Tumors in Mice Using Non-Genetically Modified Lactic Acid Bacteria Displaying E7 Antigen at Its Surface. Appl Microbiol Biotechnol (2013) 97(3):1231-9. doi: 10.1007/s00253-012-4575-1

103. Lee TY, Kim YH, Lee KS, Kim JK, Lee IH, Yang JM, et al. Human Papillomavirus Type 16 E6-Specific Antitumor Immunity Is Induced by Oral Administration of HPV16 E6-Expressing Lactobacillus Casei in C57BL/ 6 Mice. Cancer Immunol Immunother (2010) 59(11):1727-37. doi: 10.1007/ s00262-010-0903-4

104. Kawana K, Adachi K, Kojima S, Taguchi A, Tomio K, Yamashita A, et al. Oral Vaccination Against HPV E7 for Treatment of Cervical Intraepithelial Neoplasia Grade 3 (CIN3) Elicits E7-Specific Mucosal Immunity in the Cervix of CIN3 Patients. Vaccine (2014) 32(47):6233-9. doi: 10.1016/ j.vaccine.2014.09.020
105. Adachi K, Kawana K, Yokoyama T, Fuji T, Tomio A, Miura S, et al. Oral Immunization With a Lactobacillus Casei Vaccine Expressing Human Papillomavirus (HPV) Type 16 E7 Is an Effective Strategy to Induce Mucosal Cytotoxic Lymphocytes Against HPV16 E7. Vaccine (2010) 28 (16):2810-7. doi: 10.1016/j.vaccine.2010.02.005

106. Poo H, Pyo HM, Lee TY, Yoon SW, Lee JS, Kim CJ, et al. Oral Administration of Human Papillomavirus Type 16 E7 Displayed on Lactobacillus Casei Induces E7-Specific Antitumor Effects in C57/BL6 Mice. Int J Cancer (2006) 119(7):1702-9. doi: 10.1002/ijc.22035

Conflict of Interest: The authors declare that the research was conducted in the absence of any commercial or financial relationships that could be construed as a potential conflict of interest.

Publisher's Note: All claims expressed in this article are solely those of the authors and do not necessarily represent those of their affiliated organizations, or those of the publisher, the editors and the reviewers. Any product that may be evaluated in this article, or claim that may be made by its manufacturer, is not guaranteed or endorsed by the publisher.

Copyright $\odot 2021 \mathrm{Dai}, \mathrm{Du}$, Li and Wu. This is an open-access article distributed under the terms of the Creative Commons Attribution License (CC BY). The use, distribution or reproduction in other forums is permitted, provided the original author(s) and the copyright owner(s) are credited and that the original publication in this journal is cited, in accordance with accepted academic practice. No use, distribution or reproduction is permitted which does not comply with these terms. 